\title{
Coordinative control of G2/M phase of the cell cycle by non- coding RNAs in hepatocellular carcinoma
}

\author{
Jun Shi ${ }^{1}$, Guangqiang Ye ${ }^{2}$, Guoliang Zhao ${ }^{2}$, Xuedong Wang ${ }^{1}$, Chunhui Ye ${ }^{2}$, Keooudone Thammavong ${ }^{2}$, \\ Jing Xu Corresp., 2 , Jiahong Dong ${ }^{\text {Corresp. } 1}$ \\ ${ }^{1}$ Department of Hepatobiliary and Pancreas Surgery, Beijing Tsinghua Changguang Hospital, School of Clinical Medicine, Tsinghua University, Beijing, \\ China \\ 2 The First Affiliated Hospital of Guangxi Medical University, Guangxi Medical University, Nanning, China \\ Corresponding Authors: Jing Xu, Jiahong Dong \\ Email address: xujing@gxmu.edu.cn, dongjiahong@mail.tsinghua.edu.cn
}

Objective : To investigate the interaction of non-coding RNAs (ncRNAs) in hepatocellular carcinoma.

Methods : We compared the ncRNAs and mRNAs expression profiles of hepatocellular carcinoma and adjacent tissue by microarray and RT-PCR. The relationship between different ncRNAs and mRNA was analyzed using bioinformatics tools. A regulatory model of ncRNAs in HCC was developed.

Results : A total of 1704 differentially expressed IncRNAs, 57 miRNAs, and 2093 mRNAs were identified by microarray analyses. There is a co-expression relationship between two ncRNAs (miRNA-125b-2-3p and IncRNA P26302 3. Bioinformatics analysis demonstrated CDK1 and CyclinA2 as potential targets of miR-125b-2-3p and PLK1 as potential target of IncRNAP26302. All three gene are important components in the G2/M phase of cell cycle. Subsequently real-time PCR studies confirmed these microarray results.

Conclusion : MiR-125b-2-3p and IncRNAP26302 may affect the G2/M phase of the cell cycle through the regulation of their respective target genes. This study shows a role of ncRNAs in pathogenesis of hepatocellular carcinoma at molecular level, providing a basis for the future investigation aiming at early diagnosis and novel treatment of hepatocellular carcinoma. 
1 COORDINATIVE CONTROL OF G2/M PHASE OF THE

2

3

\section{HEPATOCELLULAR CARCINOMA}

4 Jun Shi ${ }^{1,}$, Guangqiang Ye ${ }^{2}$, a, Guoliang Zhao ${ }^{2}$, Xuedong Wang ${ }^{1}$, Chunhui $\mathrm{Ye}^{2}$, Keooudone

5 Thammavong ${ }^{2}$, Jing $\mathrm{Xu}^{2 *}$, Jiahong Dong ${ }^{1 *}$

$6{ }^{1}$ Department of Hepatobiliary and Pancreas Surgery, Beijing Tsinghua Changguang Hospital,

7 School of Clinical Medicine, Tsinghua University, Beijing, China

$8{ }^{2}$ The First Affiliated Hospital of Guangxi Medical University, Guangxi Medical University,

9 Nanning, China

$11{ }^{*}$ Corresponding author. E-mail addresses: xujing@gxmu.edu.cn (J XU),

12 dongjiahong@mail.tsinghua.edu.cn (JH. Dong)

$13{ }^{\text {a Co-first authors }}$

\section{【Abstract】}

17 Objective: To investigate the interaction of non-coding RNAs (ncRNAs) in

18 hepatocellular carcinoma (HCC).

19 Methods: We compared the ncRNA and mRNA expression profiles of HCC and 20 adjacent tissue by microarray and RT-PCR. The relationships between different 21 ncRNAs and mRNAs were analyzed using bioinformatics tools. A regulatory 22 model of ncRNAs in HCC was developed.

23 Results: A total of 1704 lncRNAs, 57 miRNAs, and 2093 mRNAs were identified 24 as differentially expressed in HCC by microarray analyses. A co-expression 
25 relationship was identified between two ncRNAs (miRNA-125b-2-3p and lncRNA

26 P26302). Bioinformatics analysis revealed CDK1 and CyclinA2 as potential

27 targets of miR-125b-2-3p and PLK1 as a potential target of lncRNAP26302. All

28 three genes are important components in the G2/M phase of the cell cycle.

29 Subsequently, real-time PCR studies confirmed these microarray results.

Conclusion: MiR-125b-2-3p and lncRNAP26302 may affect the G2/M phase of the cell cycle through the regulation of their respective target genes. This study demonstrates a role of ncRNAs in the pathogenesis of HCC at a molecular level, providing a basis for future investigations aimed at developing methods for early diagnosis and novel treatment of HCC.

\section{Introduction}

Hepatocellular carcinoma (HCC) is the most common primary liver cancer, accounting for $85 \%-90 \%$ of all primary livery cancer cases. Among the world's most common malignancies, the incidence of HCC is ranked 6th, and its mortality rate is ranked 3rd (Torre et al. 2015). The high mortality rate is mainly due to the strong invasive and metastatic capacity of HCC cells. Upon diagnosis, HCC is often at an advanced stage (Wang et al. 2013). Therefore, to improve the prognosis and treatment of liver cancer, investigators have been working to understand the cellular and molecular biological mechanisms leading to the malignant transformation of hepatocytes. Early diagnosis is the key to effective treatment of liver cancer. It is important to find effective early diagnosis markers of liver cancer. In recent years, a large number of studies have suggested that noncoding RNAs (ncRNA) are related to the occurrence of tumors, providing a new research direction for the diagnosis and treatment of liver cancer (Mattick \& Makunin 2006).

As more and more ncRNAs are studied, it is notable that different ncRNAs have different regulatory capabilities in the development of liver cancer. MicroRNA (miRNA) and long ncRNA (lncRNA) are the two most representative forms. For example, miR-122 is a miRNA specifically expressed in the liver and thus 
56 overexpressed in HCC cell lines HepG2 and HepB3. This miRNA promotes

57 apoptosis and inhibits proliferation of hepatoma cells in biological processes (Datta et al. 2008). In addition, miR-122 may also target the activity of p53 through the cyclin G1 gene to affect the sensitivity of HCC to doxorubicin (Hsu et al. 2012). In a study by Tsang et al. (Tsang et al. 2015), the lncRNA HOTTIP was identified as the most significantly up-regulated lncRNA in human HCCs, even in an early stage of HCC formation. Functionally, knock-down of HOTTIP attenuated HCC cell proliferation in vitro and markedly abrogated tumourigenicity in vivo. In addition, knock-down of HOTTIP also inhibited the migratory ability of HCC cells and significantly abrogated lung metastasis in an orthotopic implantation model in nude mice. Furthermore, they identified miR-125b as a post-transcriptional regulator of HOTTIP. Ectopic expression of miR-125b reduced HOTTIP-coupled luciferase activity and suppressed the endogenous level of HOTTIP. IncRNA HULC is highly expressed in HCC, while it has a binding site of miR-372. Therefore, HULC can competitively bind miRNAs, making miRNAs lose their ability to target genes, thereby reducing the tumor suppressor effect of miR-372 (Wang et al. 2010). Researchers have termed the ncRNAs that competitively bind to miRNA as competitive endogenous RNA (ceRNA) (Salmena et al. 2011). This concept has enriched the central dogma of molecular biology and provided a useful tool for analyzing the molecular mechanisms of tumors.

During the study of a single ncRNA, it was found that the effect of certain ncRNAs on tumorigenesis is accompanied by corresponding changes in other types of ncRNA. In this regard, we hypothesize that ncRNAs among different species may play a regulatory role in the development of tumors by coordinatively acting on the same target gene or upstream and downstream genes on the same signaling pathway. In order to explore whether other regulatory mechanisms of lncRNAs and miRNAs are involved in HCC pathogenesis, we compared the expression levels of lncRNA, miRNA and mRNA between HCC tissues and adjacent normal tissues. Bioinformatics prediction, correlation analysis and pathway evaluation were used to identify a potentially important ncRNA and its target genes. 
88

89 In this study, 12 pairs of primary hepatocellular carcinoma (HCC) tissues and their 90 adjacent tissues collected from $2 \mathrm{~cm}$ away from the cancer tissue were selected

91 (Chen et al. 2016). All specimens were obtained from the First Affiliated Hospital 92 of Guangxi Medical University. The tissue samples were processed within 30 93 94 95 96 97 98 99

\section{1 miRNA microarray}

112 We performed microarray analysis of miRNAs using Affymetrix GeneChip 113 miRNA arrays (Santa Clara, CA, USA). In short, polyA polymerase uses the

114 Genisphere FlashTag HSR kit to label $1 \mathrm{mg}$ of total RNA from the tissue. This was 115 followed by hybridization of RNA with Affymetrix miRNA arrays. After

116 hybridization, the standard Affymetrix ribbons were stained, washed, scanned, and 117 transformed with AGCC software (Affymetrix ${ }^{\circledR}$ GeneChip ${ }^{\circledR}$ command console ${ }^{\circledR}$ 
118 software) and the GeneChip ${ }^{\circledR}$ Scanner 3000. Using .CEL files as source files, we

119 use the Expression Console software provided by Affymetrix to perform row data 120 preprocessing: including RNA normalization, if the probe signal was significantly

121 higher than the background signal, and integration of the probe signal into a probe

122 set signal. Three miRNA microarray tests were performed on each sample.

123

$124 \operatorname{lncRNA}$ and mRNA microarray

125

126 The Jingxin ${ }^{\circledR}$ Human lncRNA + mRNA Expression Microarray (CapitalBio,

127 China) screens lncRNA and mRNA at the same time. According to the

128 manufacturer's instructions, $5 \mu \mathrm{L}$ total RNA extracted from each sample was used

129 to synthesize double-stranded complementary DNA (cDNA). Double-stranded

130 cDNA was labeled and hybridized to the Jingxin ${ }^{\circledR}$ Human lncRNA+mRNA

131 Expression Microarray. The volume of the cDNA product obtained after reverse

132 transcription and purification was concentrated to $14 \mu \mathrm{L}$, and cy3-dCTP/cy5-dCTP

133 was added and placed in a PCR machine (reaction at $37^{\circ} \mathrm{C}$ for 1.5 hours, reaction at

$13470^{\circ} \mathrm{C}$ for 5 minutes, retention at $4^{\circ} \mathrm{C}$ ). Then, the cDNA was purified using the

135 Nucleospin ${ }^{\circledR}$ Extract II (MN, Cat. No. 740609.250) kit, and the fluorescently

136 labeled product was subjected to fluorescence incorporation and nucleic acid

137 quantification using an ultraviolet spectrophotometer. Next, $100 \mu \mathrm{L}$ of the

138 hybridization solution was applied to the hybrid coverslip, and the hybridization

139 cassette was mounted on the rotor of the Hybridization Oven G2545A and

140 hybridized at $45^{\circ} \mathrm{C}$ for 12 hours. The cleaned chip was scanned with an Agilent

141 G2565CA Microarray Scanner to obtain a hybrid picture. Hybrid images were

142 analyzed and data extracted using Agilent Feature Extraction (v10.7) software.

143 Then we used Agilent GeneSpring software to normalize and analyze the data.

144

$145 \quad R T-P C R$ detection

146 Total RNA was isolated from samples using the Trizol reagent, and then cDNA

147 was synthesized using viral polymerase and random primers. The cDNA PCR 
148 products were amplified, and the target gene expression levels were normalized to

14918 s mRNA, an internal quantity control. Experiments were performed in triplicate.

150

151 Statistical analysis

152

153 We analyzed the differentially expressed genes in the preprocessed data. Three or 154 more biologically replicated data points for each sample were analyzed using the

155 SAM (significance analysis of microarray) R package (Tusher et al. 2002). The

156 screening criteria for differential genes were: q-value $\leq 5 \%$ and fold change $\geq 2$ or

$157 \leq 0.5$ (Clarke et al. 2008) (Yang et al. 2005). The genes identified as differentially

158 expressed were subjected to unsupervised hierarchical clustering (Cluster 3.0) and

159 TreeView analysis (Stanford University, Stanford, CA, USA). Moreover, gene

160 ontology (GO) enrichment analysis was carried out for differentially expressed

161 mRNAs. The array data presented in this paper have been uploaded to the NCBI

162 Gene Expression Omnibus and are accessible through GEO series accession

163 number GSE115019

164 (http://www.ncbi.nlm.nih.gov/geo/query/acc.cgi?acc=GSE115019).

165

166

167

168

Results

169

170

Differentially expressed miRNAs, $m R N A$ s, and lncRNAs in HCC

171

172 An Affymetrix miRNA microarray was used to establish the miRNA expression

173 profiles of 12 pairs of human HCC tissues and their adjacent tissues. Based on a

174 fold change of $\geq 2$ or $\leq-2$ with $\mathrm{P}<0.05$ for the t-test as the screening criteria for

175 differential genes, 57 differentially expressed miRNAs were identified, including 9 
176 that were up-regulated and 48 that were down-regulated (Figure 1A). The same

177 samples were used for gene expression profiling using the Jingxin ${ }^{\circledR}$ human

178 IncRNA+mRNA V4.0 chip. Compared to the adjacent tissue, HCC tissues showed

179 significant differences in the gene expression levels of 2093 mRNAs, among

180 which 635 were up-regulated and 1,458 were down-regulated (Figure 1B). A total

181 of 1,704 lncRNAs showed significantly different expression levels in HCC tissues,

182 of which 607 were up-regulated and 1,097 were reduced (Figure 1C). The GO

183 enrichment analysis results showed that differential mRNA sets were enriched in

184 mitotic cell cycle, DNA packaging, cell cycle phase, etc. (Figure 2).

Co-expression analysis constructs and signaling pathways

We performed a co-expression analysis of lncRNAs and miRNAs in order to select the co-expressed genes based on an absolute value of correlation coefficient $>0.7$ and $p<0.05$. The lncRNAs and miRNAs with a co-expression relationship were used to predict their target genes, which were crosschecked with genes identified by mRNA microarray analysis. We identified 20 co-expressed lncRNAs and miRNAs, of which only lncRNA P26302 had a fold difference $>10 \mathrm{X}$, so we chose miR-125-2-3p co-expressed with lncRNAP26302 (correlation coefficient $=-0.87$, $\mathrm{P}=0.0001$ ) for further study. Meanwhile, three differentially expressed mRNAs that were target genes of miR-125b-2-3p or lncRNA P26302 were identified by mRNA microarray. The target genes for miRNA-125b-2-3p were cyclin-dependent kinases 1 (CDK1) and cyclin A2. Polo-like kinase 1 (PLK1) is an target gene of lncRNA P26302. Through KEGG pathway analysis of target genes, we found that the three target genes co-exist in the G2/M phase of the cell cycle pathway, and they are close in order within the pathway. Thus, we propose the signal pathways

202 shown in Figure 3. 
206 To verify the differential expression of genes identified by microarray screening,

207 we performed RT-PCR analyses. The results showed that the expression of

208 miR125b-2-3p was significantly lower in HCC tissues than in adjacent tissues

$209\left(\mathrm{P}_{\text {miR125b-2-3p }}=0.0052\right)$, while the expression levels of lncRNAp26302, CDK1, cyclin

$210 \mathrm{~A} 2$, and PLK1 were significantly higher in HCC tissues than in adjacent tissues

$211 \quad\left(\mathrm{P}_{\mathrm{P} 26302}=0.0255 ; \mathrm{P}_{\mathrm{CCNA} 2}=0.028 ; \mathrm{P}_{\mathrm{CDK} 1}=0.0171 ; \mathrm{P}_{\mathrm{plk} 1}=0.0267\right)$. The differential

212 gene expression detected in $\mathrm{HCC}$ and adjacent tissues was consistent with the

213 results of the gene chip screening (Figure 4).

214

\section{Discussion}

216 Non-coding RNAs such as lncRNAs and miRNAs have been proven to participate

217 in the regulation of gene expression through competition for endogenous RNA

218 networks with mRNA (Kartha \& Subbaya 2014). The interaction between these

219 two ncRNAs plays an important role in tumor development (Jiang et al. 2017; Wu

220 et al. 2016). At present, the specific molecular mechanism of the interaction

221 between lncRNA and miRNA is speculated to be one of two possibilities:1.

222 because lncRNAs and mRNAs have a similar structure, a miRNA can specifically

223 bind to its 3' UTR and down-regulate lncRNA expression through a mechanism

224 similar to that of mRNA regulation (Shi et al. 2013); or 2. studies have confirmed

225 that lncRNAs can competitively target miRNAs to inhibit the expression of

226 miRNAs, thereby reducing their inhibitory effect on target genes (Salmena et al.

227 2011).

228 During the G2 to M phase transition of the cell cycle, CDKs represent a set of 229 Ser/Thr kinase systems that correspond to cell cycle progression. Various CDKs

230 are alternately activated along the cell cycle, and phosphorylation of the

231 corresponding substrate allows the cell cycle to proceed in an orderly manner.

232 Cyclin A2 is a cyclin that binds to CDK1 to form a CycA/CDK1 complex

233 (Santamaría et al. 2007). The CycA/CDK1 complex is the main damage

234 monitoring mechanism in $\mathrm{S}$ phase. The withdrawal of the cell cycle from mitosis

235 requires CDK1 inactivation, and the most important mechanism of CDK1

236 inactivation is the hydrolysis of mitotic cyclins. In higher eukaryotes, this involves

237 the continuous destruction of type A and type B cyclins, which results in 
238 successive inactivation of the $\mathrm{CycA} / \mathrm{CDK} 1$ complex and the $\mathrm{CycB} / \mathrm{CDK} 1$

239 complex. When DNA damage occurs, Cyclin A is first destroyed, resulting in the 240 inactivation of CycA/CDK1 necessary for the G2 to M transition (Kaspar et al.

241 2001). Finally, the cell cycle is stopped in the G2 phase. PLK1 represents a class of

242 highly conserved serine/threonine protein kinases expressed in eukaryotes (Takaki

243 et al. 2008). It has been proven in many studies that Plkl is a key gene in cell cycle 244 regulation and is regulated by phosphorylation and protein degradation (Barr et al. 245 2004) (Catherine \& Jonathon 2004). Current studies have found that PLK1 is able 246 to restart mitosis by acting on the CycA/CDK1 complex, from the $\mathrm{G} 2$ phase to the $247 \mathrm{M}$ phase. In the $\mathrm{G} 2$ phase, the cytokine $\mathrm{CycA} / \mathrm{CDK} 1$ complex is inactivated due to 248 the phosphorylation of the residues on the adenosine triphosphate-binding domain of Cdk1, while Myt1 kinase phosphorylates the threonine residue Tyr15. This stops entrance into the mitosis phase before DNA replication is completed and destroys genome integrity (Takizawa \& Morgan 2000). Plk1 is able to phosphorylate and inhibit the activation of Myt1, resulting in dephosphorylation of CDK1, and activation of CycA/CDK1 complex initiates mitosis (Nakajima et al. 2003).

To further explore the relationship among the three groups, we performed co-

255

256

257

258

259

260

261

262

263

264

265

266

267

268

269

270

271 expression analysis of the differentially expressed miRNAs and lncRNAs and analyzed the KEGG enrichment pathways of their respective target genes. We found that miRNA125b-2-3p and lncRNAP26302 expression levels correlated in HCC. Moreover, the target genes CDK1 and cyclin A2 of miR-125b-2-3p are in the same gene pathway (the G2/M phase of the cell cycle) and of the target gene PLK1 of 1ncRNAP26302. In the normal cell cycle, CycA/CDK1 is the major damage regulation mechanism of S phase (Katsuno \& Mak 2009), and cyclin A2 in CycA can promote the synthesis of DNA in S phase, thus promoting the transition of cells from the G2/M phase into M phase (Nikola et al. 2012). However, when DNA damage occurs, cell cycle regulation is influenced by the DNA damage repair mechanism, which inhibits the expression of CycA/CDK1 through the Myt1 gene (Varadarajan et al. 2016), thus allowing the cell cycle to stay in the $\mathrm{S}$ phase for cell repair (Chapman et al. 2012; Inger \& Gent 2012). In hepatoma cells, the target gene PLK1 of lncRNAP26302 was overexpressed, inhibiting the expression of Myt1 and reducing the inhibitory effect of Myt1 on CycA/CDK1. As a result, when DNA damage or mutation occurs in the nuclei of tumor cells, the chromosomes can bypass the DNA damage detection point, and these cells 
272 continue to proliferate and divide, thereby promoting the formation of HCC 273 (Perdiguero \& Nebreda 2004). In addition, chip detection revealed that low 274 expression of miR-125b-2-3p leads to an increase in the expression of the target 275 gene cyclin A2, further promoting the formation of CycA/CDK1. By differential

276

277

278

279

280

281

282

283

284

285

286

287

288

289

290

291

292

293

294

295

296

297

298

299

300

301

302

gene correlation analysis, a reverse correlation was found between miR-125b-2-3p and lncRNAP26302, which indicates that the decreased expression of miR-125b-2$3 p$ in HCC may cause overexpression of lncRNAp26302. Therefore, miR-125b-2$3 p$ and lncRNAP26302 may have a coordinative effect on this pathway, together promoting the expression of $\mathrm{CycA} / \mathrm{CDK} 1$, so as to coordinatively promote the formation of liver cancer.

We also performed RT-PCR validation of the differential expression of the genes regulated by the identified lncRNAs. Among them, the RT-PCR results for hs-mir125b-2-3p, lncRNAp26302(uc003lzi.3), CDK1, cyclin A2 and PLK1 were consistent with their expression in the G2/M phase of the cell cycle. Therefore, we believe that miRNA 125b-2-3p and lncRNA p26302 have a coordinative regulatory effect on the $\mathrm{G} 2 / \mathrm{M}$ phase in $\mathrm{HCC}$.

\section{Conclusions}

In summary, a large number ncRNAs are differentially expressed between HCC tissue and adjacent normal tissue. From the expression profiling study of these genes, we propose that mir-125b-2-3p and lncRNA p26302 affect the G2/M phase of the cell cycle through a coordinative regulation of their respective target genes. The gene expression results of this study expand our understanding of the gene expression mechanism of HCC, providing support for the ceRNA hypothesis and a more complete understanding of the role of ncRNAs in the occurrence and development of HCC and the regulatory loop. In the future, functional cell cycle analysis is needed to demonstrate the role of miR-125b-2-3p and lncRNAP26302 target genes in the cell cycle $\mathrm{G} 2 / \mathrm{M}$ phase. 


\section{References}

304 Barr FA, Silljé HHW, and Nigg EA. 2004. Polo-like kinases and the orchestration of cell division. Nat Rev Mol Cell Biol 5:429-440.

306 Catherine L, and Jonathon P. 2004. Ordered proteolysis in anaphase inactivates Plk1 to contribute to proper mitotic exit in human cells. Journal of Cell Biology 164:233-241.

Chapman JR, Taylor MR, and Boulton SJ. 2012. Playing the End Game: DNA Double-Strand Break Repair Pathway Choice. Molecular Cell 47:497-510. Cancer statistics in China, 2015. CA Cancer J Clin 66:115-132. 10.3322/caac.21338

Clarke R, Ressom HW, Wang A, Xuan J, Liu MC, Gehan EA, and Wang Y. 2008. The properties of high-dimensional data spaces: implications for exploring gene and protein expression data. Nature Reviews Cancer 8:37-49.

Datta J, Kutay H, Nasser MW, Nuovo GJ, Wang B, Majumder S, Liu CG, Volinia S, Croce CM, and Schmittgen TD. 2008. Methylation Mediated Silencing of MicroRNA-1 Gene and Its Role in Hepatocellular Carcinogenesis. Cancer Research 68:5049.

Hsu S, Wang B, Kota J, Yu J, Costinean S, Kutay H, Yu L, Bai S, Perle KL, and Chivukula RR. 2012. Essential metabolic, anti-inflammatory, and anti-tumorigenic functions of miR-122 in liver. Journal of Clinical Investigation 122:2871-2883.

Inger B, and Gent DC. 2012. Pathway choice in DNA double strand break repair: observations of a balancing act. Genome Integrity 3:9-9.

Jiang H, Ma R, Zou S, Wang Y, Li Z, and Li W. 2017. Reconstruction and analysis of the lncRNA-miRNA-mRNA network based on competitive endogenous RNA reveal functional lncRNAs in rheumatoid arthritis. Molecular Biosystems 13:1182.

Kartha RV, and Subbaya S. 2014. Competing endogenous RNAs (ceRNAs): new entrants to the intricacies of gene regulation. Frontiers in Genetics 5:8.

Kaspar M, Dienemann A, Schulze C, and Sprenger F. 2001. Mitotic degradation of cyclin A is mediated by multiple and novel destruction signals. Current Biology Cb 11:685-690.

Katsuno Y, and Mak TW. 2009. Cyclin A-Cdk1 regulates the origin firing program in mammalian cells. Proceedings of the National Academy of Sciences of the United States of America 106:3184. $1: R 17$. 
335 Nakajima H, Toyoshima-Morimoto F, Taniguchi E, and Nishida E. 2003. Identification of a

336

337

338

339

340

341

342

343

344

345

346

347

348

349

350

351

352

353

354

355

356

357

358

359

360

361

362

363

364

365

366

367 consensus motif for Plk (Polo-like kinase) phosphorylation reveals Myt1 as a Plk1 substrate. Journal of Biological Chemistry 278:25277-25280.

Nikola A, Nawal B, Marion P, Christina BP, Cosette R, Gilles G, Nathalie B, Frédéric B, Bénédicte L, and Marie BJ. 2012. A novel function for Cyclin A2: Control of cell invasion via RhoA signaling. Journal of Cell Biology 196:147.

Perdiguero E, and Nebreda AR. 2004. Regulation of Cdc25C activity during the meiotic G2/M transition. Cell Cycle 3:733-737.

Salmena L, Poliseno L, Tay Y, Kats L, and Pandolfi PP. 2011. A ceRNA hypothesis: the Rosetta stone of a hidden RNA language? Cell 146:353-358.

Santamaría D, Barrière C, Cerqueira A, Hunt S, Tardy C, Newton K, Cáceres JF, Dubus P, Malumbres M, and Barbacid M. 2007. Cdk1 is sufficient to drive the mammalian cell cycle. Nature 448:811-815.

Shi X, Sun M, Liu H, Yao Y, and Song Y. 2013. Long non-coding RNAs: a new frontier in the study of human diseases. Cancer Letters 339:159-166.

Taki Takaki T, Trenz K, Costanzo V, and Petronczki M. 2008. Polo-like kinase 1 reaches beyond mitosis--cytokinesis, DNA damage response, and development. Current Opinion in Cell Biology 20:650-660. zawa CG, and Morgan DO. 2000. Control of mitosis by changes in the subcellular location of cyclin-B1-Cdk1 and Cdc25C. Current Opinion in Cell Biology 12:658-665.

Torre L, Bray F, Siegel R, Ferlay J, Lortettieulent J, and Jemal A. 2015. Global cancer statistics, 2012: Global Cancer Statistics, 2012. Ca A Cancer Journal for Clinicians 65:87-108.

Tsang FHC, Au SLK, Wei L, Fan DNY, Lee JMF, Wong CCL, Ng IOL, and Wong CM. 2015. Long non-coding RNA HOTTIP is frequently up-regulated in hepatocellular carcinoma and is targeted by tumour suppressive miR-125b. Liver International 35:1597-1606.

Tusher VG, Tibshirani R, and Chu G. 2002. Significance analysis of microarrays. US. p 51165121.

Varadarajan R, Ayeni J, Jin Z, Homola E, and Campbell SD. 2016. Myt1 inhibition of Cyclin $\mathrm{A} / \mathrm{Cdk} 1$ is essential for fusome integrity and pre-meiotic centriole engagement in Drosophila spermatocytes. Molecular Biology of the Cell 27:2051.

Wang J, Liu X, Wu H, Ni P, Gu Z, Qiao Y, Chen N, Sun F, and Fan Q. 2010. CREB upregulates long non-coding RNA, HULC expression through interaction with microRNA372 in liver cancer. Nucleic Acids Research 38:5366-5383. 
368 Wang X, Lin S, Yu I, Qiu H, Lan Y, and Yano E. 2013. Cause-specific mortality in a Chinese 369 chrysotile textile worker cohort. Cancer Science 104:245-249.

370 Wu Q, Guo L, Jiang F, Li L, Li Z, and Chen F. 2016. Analysis of the miRNA-mRNA-lncRNA 371 networks in ER+ and ER- breast cancer cell lines. Journal of Cellular \& Molecular Medicine 19:2874-2887.

373 Yang H, Crawford N, Lukes L, Finney R, Lancaster M, and Hunter KW. 2005. Metastasis

374 predictive signature profiles pre-exist in normal tissues. Clinical \& Experimental Metastasis 22:593-603.

376

377 
Figure 1

The result of cluster analysis

(A) miRNA cluster analysis; (B) IncRNA cluster analysis; (C) mRNA cluster analysis.

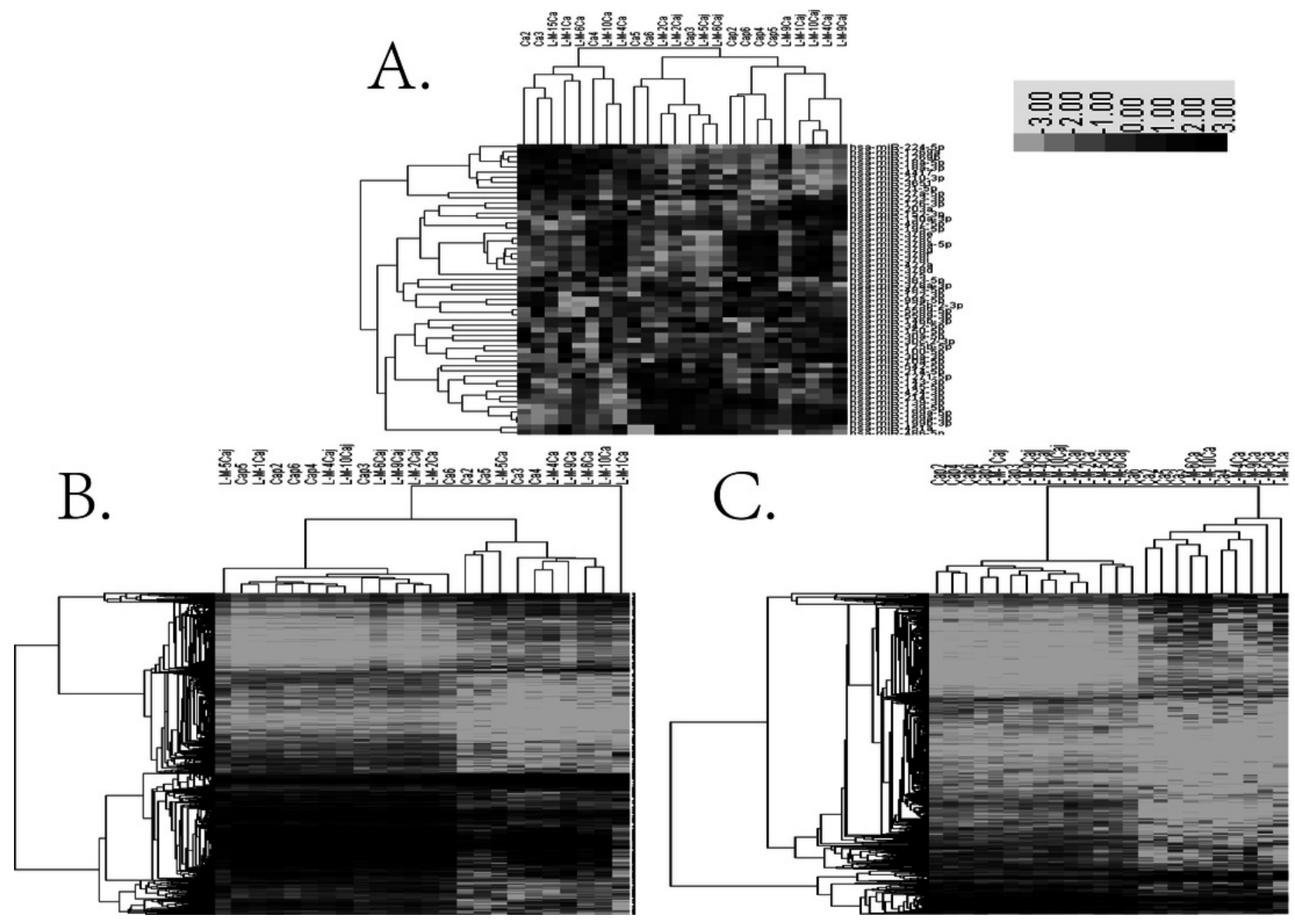


Figure 2

The result of Go enrichment analysis

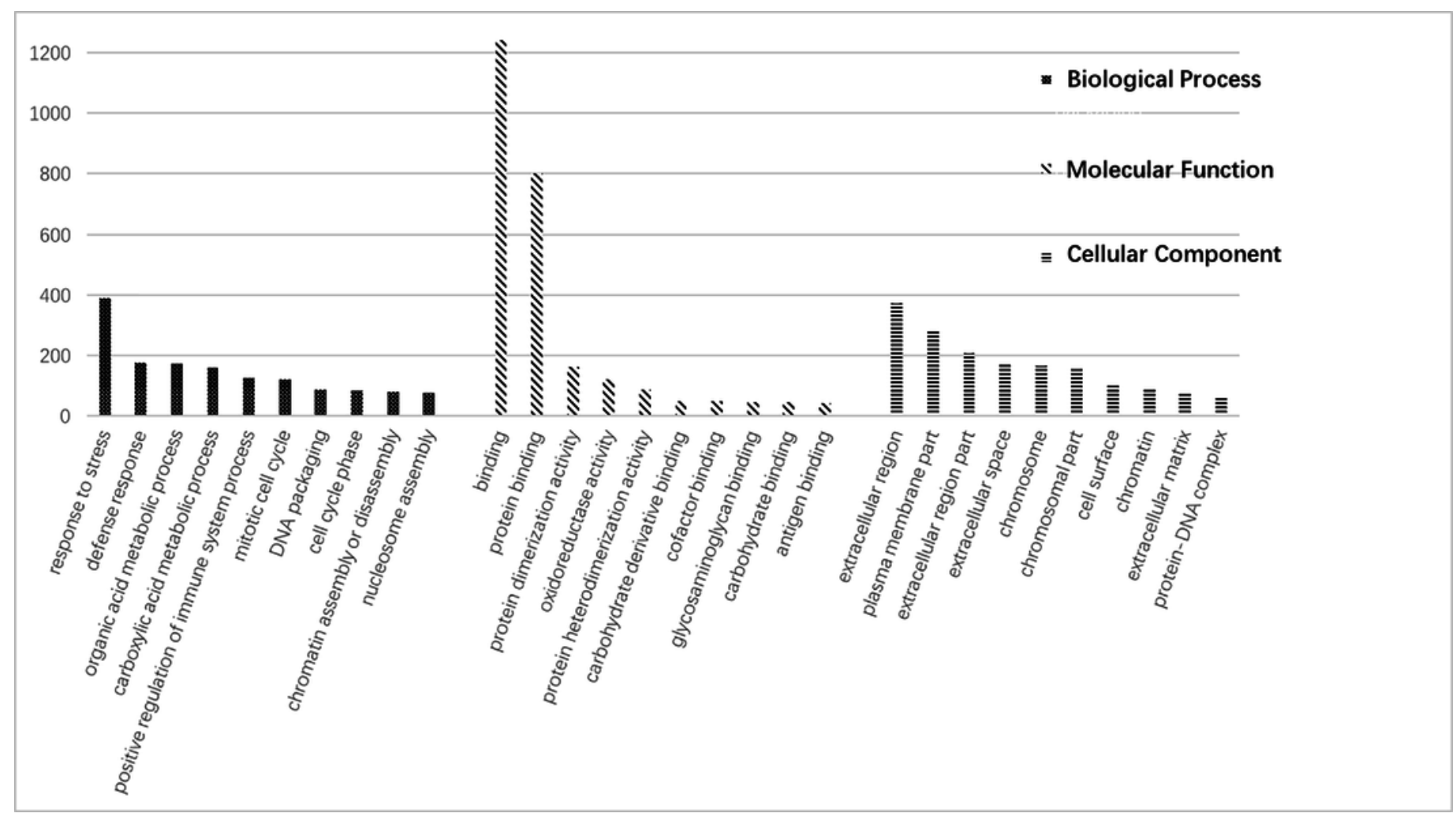


Figure 3

Simultaneous control of non-coding RNA on the G2/M phase of the cell cycle in hepatocellular carcinoma cells

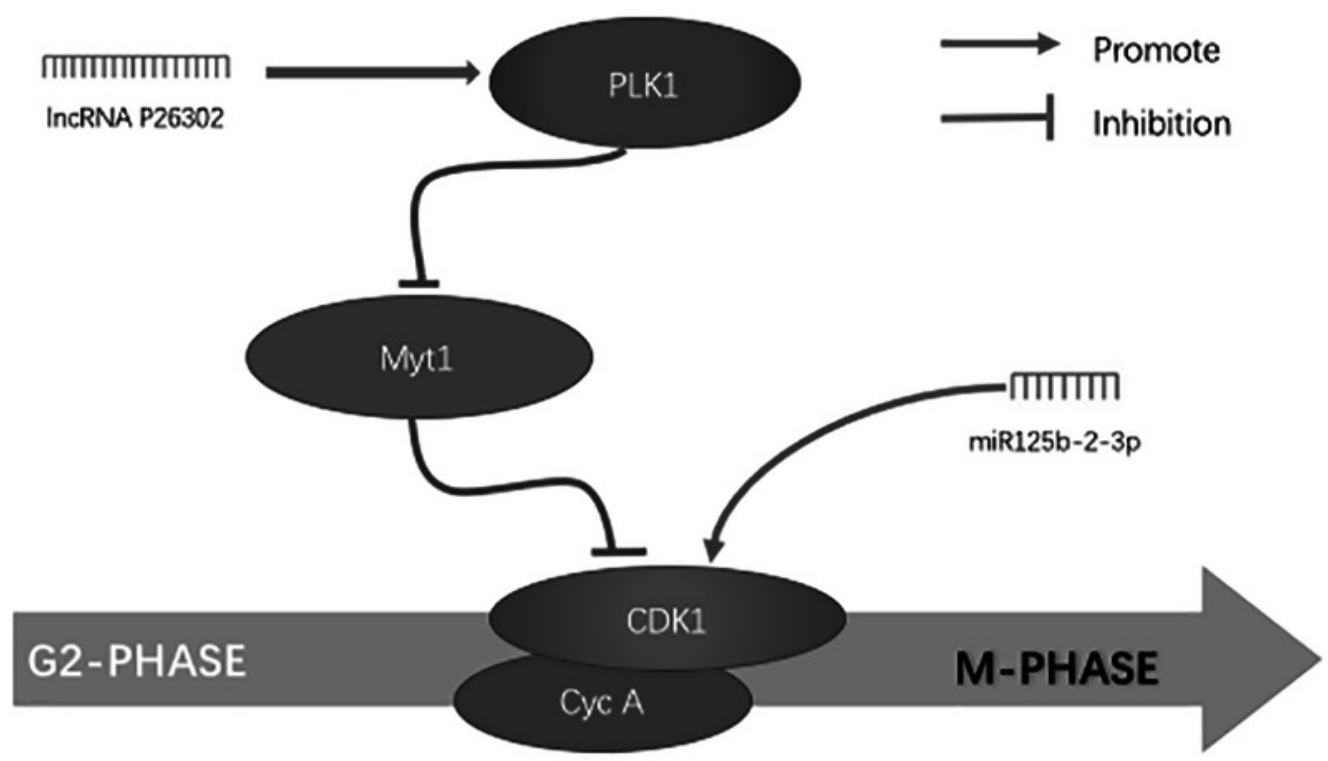


Figure 4

The results of differential gene RT-PCR

(A) $\mathrm{P}_{\text {mir125b-2-3p }}=0.0052 ;$ (B) $\mathrm{P}_{\mathrm{P} 26302}=0.0255 ;$ (C) $\mathrm{P}_{\mathrm{CCNA} 2}=0.028$; (D) $\mathrm{P}_{\mathrm{CDK} 1}=0.0171 ;(\mathrm{E}) \mathrm{P}_{\mathrm{plk} 1}=0.0267$
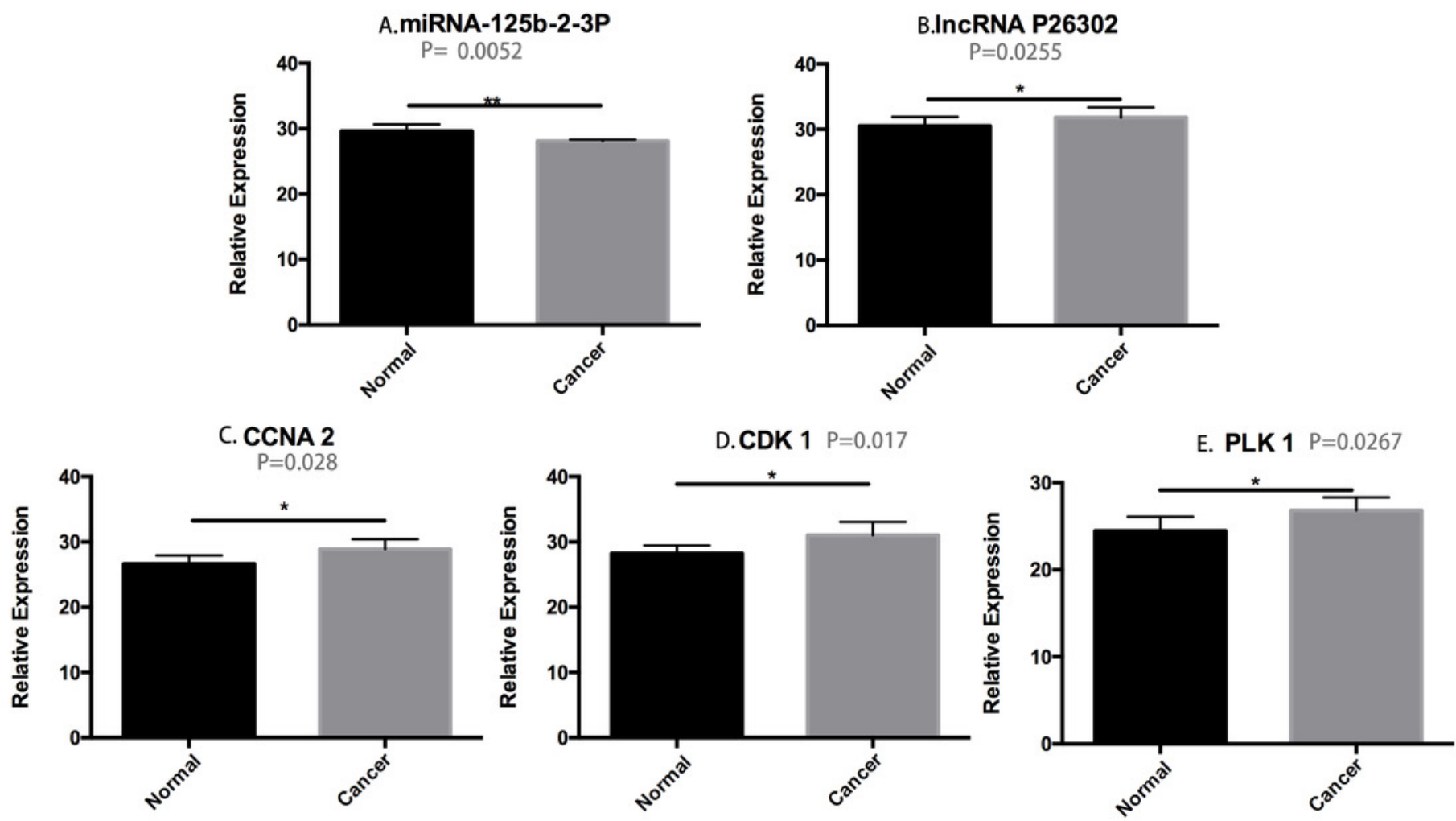